\title{
tic\&société
}

Vol. 12, $\mathrm{N}^{\circ} 2$ | 2ème semestre 2018

Numérique et situations de handicap : les enjeux de l'accessibilité

\section{Penser le numérique au prisme des situations de handicap : enjeux et paradoxes de l'accessibilité}

Nathalie PINÈDE

\section{(2) OpenEdition \\ Journals}

Édition électronique

URL : http://journals.openedition.org/ticetsociete/2573

DOI : 10.4000/ticetsociete.2573

Éditeur

Association ARTIC

Édition imprimée

Pagination : 9-43

Référence électronique

Nathalie PINEDE, « Penser le numérique au prisme des situations de handicap : enjeux et paradoxes de l'accessibilité », tic\&société [En ligne], Vol. 12, № 2 I 2ème semestre 2018, mis en ligne le 10 décembre 2018, consulté le 01 mai 2019. URL : http://journals.openedition.org/ticetsociete/2573 ; DOI : 10.4000/ticetsociete. 2573 
tic\&société - 12(2), 2018

\title{
Penser le numérique au prisme des situations de handicap : enjeux et paradoxes de l'accessibilité
}

\begin{abstract}
NATHALIE PINÈDE
Nathalie Pinède est maître de conférences en sciences de l'information et de la communication à I'IUT Bordeaux Montaigne et chercheur au laboratoire MICA (Médiation, Information, Communication et Arts- EA 4426), dans l'axe E3D (Études digitales, des données aux dispositifs). Ses travaux portent sur l'analyse des dispositifs info-communicationnels numériques, des stratégies aux usages. Elle co-dirige actuellement (avec Véronique Lespinet-Najib) un projet de recherche interdisciplinaire intitulé «Fractures corporelles, fractures numériques: enjeux, risques, solutions » (financé par la Maison des Sciences de l'Homme d'Aquitaine), projet dans lequel les questions en rapport avec l'accessibilité numérique sont centrales. nathalie.pinede@ubordeaux-montaigne.fr
\end{abstract}


Penser le numérique au prisme des situations de handicap : enjeux et paradoxes de l'accessibilité

\section{Penser le numérique au prisme des situations de handicap : enjeux et paradoxes de l'accessibilité}

Résumé : Les situations de handicap représentent un enjeu social majeur, qui interroge les facettes multiples de notre environnement et les restrictions de participation pouvant en découler. Nous explorons dans ce texte l'articulation numérique/handicap, notamment en tant que posture ambivalente entre inclusion et exclusion. Afin de limiter les risques de fractures et d'inégalités numériques, l'accessibilité des sites web, des services en ligne et d'autres applications mobiles devient un enjeu déterminant, pour lequel un arsenal normatif, réglementaire, technique, éducatif existe. Néanmoins, dans la mise en œuvre de l'accessibilité numérique, que l'on peut appréhender comme instrument de médiation, demeurent encore nombre de réticences, de méconnaissances et de difficultés. Mais des effets paradoxaux apparaissent également, en rapport avec les injonctions positives à une forme d'inéluctabilité du monde numérique.

Mots-clés : accessibilité, enjeux, fractures numériques, handicap, lois, médiation, normes, numérique, paradoxes, société.

Abstract: Disability situations are a major social issue, one that raises questions about the various aspects of our environment and restrictions on social participation that may result. In this text, we explore the link between digital and disability, highlighting its ambivalence between inclusion and exclusion. In order to limit the risks of digital divides and inequalities, the accessibility of websites, online services and other mobile applications is becoming a determining perequisite, strengthened by numerous normative, regulatory, technical and educational tools. Nevertheless, the implementation of digital accessibility, understood as an instrument of mediation, is still fraught with many reservations, misunderstandings and difficulties. Other paradoxes can also appear in connection with positive injunctions concerning the inevitability of a digital world. 


\section{Nathalie PINÈDE}

Keywords: accessibility, issues, digital divides, disability, laws, mediation, standards, digital, paradoxes, society.

Resumen: Las situaciones de discapacidad representan un problema social importante, que cuestionan las múltiples facetas de nuestro entorno y las dificultades que pudieran existir respecto a la participación. En este texto se explora la articulación digital/discapacidad/, en tanto que postura ambivalente entre inclusión y exclusión. Para limitar los riesgos de fracturas y de desigualdades digitales, la accesibilidad a los sitios web, servicios en línea y otras aplicaciones móviles constituyen una cuestión esencial, $y$, en consecuencia, existe un arsenal normativo, reglamentario, técnico y educativo. Sin embargo, en la implementación de la accesibilidad digital, que puede entenderse como un instrumento de mediación, existen aún muchas reticencias, malentendidos y dificultades. A la vez, también existen aparecen ciertas paradojas, en conexión con los preceptos positivos que plantean la inevitabilidad del mundo digital.

Palabras clave: accesibilidad, retos, fractura digital, discapacidad, leyes, mediación, normas, digital, paradojas, sociedad. 
Penser le numérique au prisme des situations de handicap : enjeux et paradoxes de l'accessibilité

\section{Introduction}

Derrière le terme numérique, mot "passe-partout " (VitaliRosati, 2014), se dessine une réalité où celui-ci, dans ses innombrables formes, s'impose comme une composante pleine et entière de nos activités. L'une des évolutions (révolutions?) majeures liées à cette convergence massive des contenus, des services et des données sur des supports en lien aux techniques du numérique consiste effectivement en ces effets d'immixtion dans les sillons multiples de nos vies, que cela relève des domaines professionnel, social, privé ou encore des loisirs. Le passage par le numérique devient dès lors une pratique quasi permanente. Celle-ci peut relever d'un choix, mais elle s'impose aussi dans bien des cas comme une nécessité, par exemple dans les environnements professionnels ou encore dans ses démarches de citoyen, en confrontant l'individu à la dématérialisation toujours plus grande de procédures, notamment administratives.

Qu'en est-il pour ceux qui se trouvent dans une situation de handicap ? Au cœur des techniques connectées se logent des formes d'implicite, révélatrices d'enjeux et de contradictions: implicite de la capacité de manipulation (d'un objet tangible), implicite de l'accès aux contenus et aux savoirs, avec les procédures cognitives qui l'accompagnent. La problématique du handicap se pose au regard de la dimension "scénarisée " de l'objet technique, le script (au sens d'Akrich, 2010), les contraintes d'organisation et d'architecture d'un document numérique, mais aussi les affordances des interfaces. Tous ces éléments peuvent entrer en conflit avec des spécificités sensorielles (vue, toucher, ouïe), mais aussi cognitives, vis-à-vis desquelles, en tant qu'individus, nous ne sommes pas tous égaux. Ainsi, il est évident que le toucher est un sens extrêmement mobilisé aujourd'hui dans notre rapport au numérique, notamment sur les smartphones et autres tablettes. Or ce geste "naturel » que nous apprécions tant sur les interfaces tactiles, cette dimension "pro-polysensorielle ", impliquant le «corps avec le sens du toucher qui se fait ici doux, feutré, intime ", reposant sur le "pari de l'intuitivité " (Saemmer, 


\section{Nathalie PINÈDE}

2017, p. 65), ce geste-là ne peut se réaliser de la façon initialement prévue lorsque le corps présente certaines altérations limitant l'activation de cette fonctionnalité.

Comment, dès lors, envisager le rapport au numérique, quand existent des difficultés sur le plan physique, sensoriel, ou encore cognitif ? Quels dangers et quelles occasions se dessinent? En quoi l'accessibilité numérique se pose-t-elle comme l'un des remèdes possibles pour la problématique du handicap, s'inscrivant en tant que médiation et remédiation, dans un jeu complexe qui n'est pas exempt de paradoxes ? Ce sont ces aspects que nous explorerons dans ce texte, à travers un panorama global des enjeux, interrogés du point de vue de la problématique, singulière mais à résonnance universelle, du handicap.

\section{Handicap et numérique : une vision pharmacologique}

Penser le numérique au double prisme du handicap (au sens large du terme) et de l'accessibilité renvoie à des problématiques complexes qui entrelacent plusieurs dimensions. C'est tout d'abord une question sociétale qui se pose. $\mathrm{Si}$, ainsi que nous l'avons vu précédemment, les enjeux sociaux majeurs relatifs au numérique apparaissent aisément, en est-il de même pour les questions liées au handicap ? Avant toute chose, il est intéressant de rappeler ou de préciser à quel point la qualification du handicap, de I' " infirme " dans les années 1930 à la " personne en situation de handicap " en 2005, a évolué au fil du temps, non sans conséquences sur les modes d'action publique. Les transformations en matière de classification et de dénomination du handicap illustrent bien les dynamiques de ces représentations sociétales (Lespinet-Najib et Bélio, 2013). En 1980, la Classification internationale du handicap $(\mathrm{ClH})$ de l'Organisation mondiale de la santé (OMS) propose des entrées par les déficiences, les incapacités ou les désavantages, soit principalement en rapport avec la maladie et la santé. Vingt ans plus tard, en 2001, l'OMS propose une nouvelle classification, la Classification internationale du fonctionnement, du handicap et de 
Penser le numérique au prisme des situations de handicap : enjeux et paradoxes de l'accessibilité

la santé $(\mathrm{CIF})^{1}$, qui dépasse une entrée par les seules déficiences pour prendre en compte de façon multidimensionnelle la situation de la personne, en rapport avec des facteurs environnementaux et personnels, ou encore avec le contexte d'activités dans lequel elle évolue. La bascule s'opère ainsi d'une représentation de la personne dite "handicapée " (responsabilité individuelle) à une représentation de la personne en situation de handicap (responsabilité environnementale et donc sociale).

Sur le plan réglementaire, en France, la loi du 11 février 2005, en assimilant le handicap à «toute limitation d'activité ou restriction de participation à la vie en société en raison d'une altération substantielle, durable ou définitive d'une ou plusieurs fonctions physiques, sensorielles, mentales, cognitives ou psychiques, d'un polyhandicap ou d'un trouble de santé invalidant ${ }^{2}$ ", confirme ce changement de regard et ce transfert d'une responsabilité individuelle à une responsabilité collective, en rapport avec l'environnement de la personne. Cette vision du handicap élargit ainsi le périmètre en renvoyant à des situations pérennes ou ponctuelles, natives ou soudaines, ainsi qu'à des vulnérabilités ou des fragilités pouvant s'installer avec l'âge. Les seniors sont en effet particulièrement touchés par des modifications sensorielles et cognitives importantes, et le phénomène de vieillissement important de la population, notamment dans les pays industrialisés, accroît la place de ces nouvelles formes reconnues de handicap.

Pour autant, évaluer quantitativement le nombre de personnes concernées par un ou plusieurs handicaps reste un exercice délicat, et les chiffres qui circulent sont parfois datés ou peu renseignés sur les modalités d'investigation. Roy (2016) rappelle qu'en France, la dernière grande enquête de I'INSEE sur la thématique "handicap et santé " date de 2008-2009 (Bouvier,

\footnotetext{
${ }^{1}$ Disponible sur http://apps.who.int/iris/handle/10665/42418. Cette nouvelle classification s'inspire de Patrick Fougeyrollas et al. (1998) et de son modèle systémique de "Processus de production de handicap » (PPH).

${ }^{2}$ Loi 2005-102 du 11 février 2005 sur « l'égalité des droits et des chances, la participation et la citoyenneté des personnes handicapées ». Disponible sur le site Legifrance :

https://www.legifrance.gouv.fr/affichTexte.do?cidTexte=JORFTEXT000000809647\&categ orieLien=id.
} 


\section{Nathalie PINÈDE}

2011). Dans ce cadre, ce sont en France environ 11,5 millions de personnes, âgées d'au moins 16 ans, qui sont identifiées comme étant handicapées selon la base de l'un ou l'autre de ces trois critères: impossibilité ou grande difficulté physique/cognitive; restriction importante ou absolue dans la pratique des activités quotidiennes ; forte limitation due à un problème de santé depuis au moins six mois dans les activités quotidiennes. L'OMS, quant à elle, annonce que plus d'un milliard de personnes, soit environ $15 \%$ de la population, vivraient avec une forme ou une autre de handicap, et cette proportion liée au handicap aurait tendance à augmenter, notamment du fait du vieillissement de la population et de la fréquence croissante des maladies chroniques ${ }^{3}$. Pourtant, malgré ces chiffres impressionnants, une forme d'invisibilité demeure du point de vue social : en effet, si le handicap moteur est emblématique du handicap en général, car le plus "spectaculaire " dans sa forme visible, $80 \%$ des handicaps seraient invisibles (handicaps auditif et psychique, maladies chroniques par exemple).

Handicap et numérique posent donc chacun des enjeux sociaux d'importance et l'articulation handicap/numérique révèle d'une part des possibilités d'ouverture et de décloisonnement et, d'autre part, des restrictions liées à la fausse " évidence " du numérique. Cette articulation s'inscrit fondamentalement dans une logique ambivalente, entre effets curatifs et toxiques, logique que l'on peut qualifier de "pharmacologique ", si l'on reprend la lecture du numérique proposée par Stiegler $(2010)^{4}$. Cette ambivalence est notamment soulignée par Casilli (2010), lorsqu'il distingue les "technologies capacitantes" (enabling technologies) des technologies « incapacitantes » (disabling technologies). Du côté des "technologies capacitantes », permettant de suppléer à certaines limitations relatives aux types de handicap (moteur, par exemple), on peut évoquer la facilitation d'accès à des ressources informationnelles et communicationnelles, ou encore la possibilité

\footnotetext{
${ }^{3}$ Source : http://www.who.int/fr/news-room/fact-sheets/detail/disability-and-health. Voir aussi le Rapport mondial sur le handicap, disponible sur: http://www.who.int/disabilities/world report/2011/report/fr/.

${ }^{4}$ "Tout objet technique est pharmacologique : il est à la fois poison et remède » (Vocabulaire Ars Industrialis : http://www.arsindustrialis.org/pharmakon).
} 
Penser le numérique au prisme des situations de handicap : enjeux et paradoxes de l'accessibilité

de développer une identité performative en ligne, ce qui contribue à favoriser l'intégration sociale. Dans le cas de personnes en situation de handicap psychique, Ayon (2016) positionne le numérique à partir d'activités coopératives communes, "comme environnement inclusif » (p. 165).

A contrario, les injonctions - explicites ou implicites - d'accès aux environnements numériques (pour l'insertion professionnelle, les démarches administratives, etc.), mais aussi le simple désir légitime - de participer à ces univers, peuvent se heurter au mur des outils et de la conception des services, entraînant dès lors des possibilités de marginalisation ainsi que le renforcement des vulnérabilités (disabling technologies). Dès lors, le risque de disability divide (fracture par le handicap [Dobransky et Hargittai, 2006]) n'est pas mince, nous renvoyant à une double fracture numérique, d'une part, entre "les usagers des TIC qui sont handicapés et ceux qui ne sont pas handicapés et d'autre part, les handicapés usagers des TIC et ceux qui ne sont pas usagers des TIC » (Casilli, 2010, p. 503). L'entrée par les fractures numériques s'avère donc une entrée pertinente permettant de décrypter certains risques pour les personnes en situation de handicap.

\section{Fractures et inégalités numériques liées au handicap}

À partir d'une revue de la littérature sur le sujet, on peut identifier trois types de fractures numériques: instrumentale, méthodologique (second order digital divide) et sociale. La fracture dite "instrumentale » met au premier rang la question de l'équipement et du matériel ; elle est souvent envisagée à travers le " fameux " axe Nord-Sud, mais aussi à travers des oppositions de type rural/urbain. Dans ce premier niveau de lecture, l'approche purement quantitative est dominante : il s'agit là de combler le manque en équipements et en accès (accès au réseau Internet, à la téléphonie mobile, etc.) pour rendre possible l'accès à l'information. La recherche d'indicateurs multiples, la production de statistiques pour évaluer les taux d'équipements, de connexions, etc., battent ici leur plein. 


\section{Nathalie PINÈDE}

Cette dimension instrumentale de la fracture numérique présente l'avantage d'être visible, partiellement transparente, en tout cas identifiable et mesurable à l'aide d'indicateurs variés (Soupizet, 2004). De fait, les écarts en matière d'équipements n'ont cessé de s'amenuiser sensiblement, la volonté politique étant là, mais sans que disparaissent pour autant nombre d'inégalités relatives au numérique (Lamarche, Rallet et Zimmermann, 2006). Ainsi, à un niveau macro, les indicateurs peuvent dénoter une augmentation des taux d'équipement, tout en masquant, à un degré d'analyse affiné, des différenciations notables, voire le renforcement de certaines inégalités (George, 2004). II demeure donc illusoire d'espérer l'équité par un utopique alignement des indicateurs d'équipement, les moyens d'accès matériel représentant une condition nécessaire mais non suffisante.

Autre type de fracture numérique, celle dite de "second degré » (second order digital divide) renvoie " aux compétences requises des utilisateurs pour qu'ils puissent réellement s'approprier les contenus de l'information et des services en ligne, voire devenir eux-mêmes offreurs d'informations ou de services " (Vendramin et Valenduc, 2006, p. 143). Des carences sur le plan de la maîtrise de certains savoirs et de connaissances relevant d'une culture informationnelle (Rizza, 2006), mais aussi de savoir-faire en matière de méthode constituent un obstacle supplémentaire à un indispensable processus (procès) d'appropriation technologique ${ }^{5}$. Cela suppose la conjonction de nombreux facteurs, auxquels participent les processus de médiation (humaine, sociale, culturelle). L'aisance dans la manipulation technique ne garantit pas de facto une qualité du point de vue des résultats obtenus ou une optimisation sensible de la démarche d'appropriation cognitive. Ces alliances subtiles entre les possibilités ouvertes sur un plan instrumental et leur réelle appropriation par réinvestissement dans des stratégies diversifiées sont susceptibles de créer des phénomènes de rupture plus ou moins prononcés au sein des dynamiques d'apprentissage. Ces compétences multiples, reflets d'une "littératie numérique » (digital literacy [Buckingham, 2016 ; Gilster, 1997]), inégales selon les individus,

\footnotetext{
${ }^{5}$ Ce qui renvoie notamment aux risques d'une population de jeunes « sur-équipée », mais pouvant être fragile dans son rapport à l'information et à la communication.
} 
Penser le numérique au prisme des situations de handicap : enjeux et paradoxes de l'accessibilité

les outils mobilisés ou encore les parcours sont autant de fêlures possibles dans les parcours au cœur du numérique.

D'autres facteurs, d'ordre social, peuvent enfin être identifiés. En effet, la frontière inclusion/exclusion sociale est loin de disparaître avec la banalisation et l'extension des réseaux. Le concept d'individualisme connecté exploré par Patrice Flichy (2004) illustre bien ce risque permanent de décrochage à deux niveaux, social et technologique: "Les TIC peuvent offrir de nouveaux moyens pour renforcer l'autonomie et les contacts, en un mot "l'affiliation"; [...] mais dans beaucoup de cas, elles vont renforcer la désaffiliation des désaffiliés. La fracture numérique renforce la fracture sociale » (p. 46). Cela peut même prendre des formes encore plus diffuses et pernicieuses, renvoyant à notre relation à autrui et à notre place dans la société.

Disposer des infrastructures, des compétences minimums et construire des répertoires d'usages stables ne sauraient être présentés comme les signes explicites d'un dépassement de la fracture numérique. Car celle-ci [...] étend son spectre sur la mal-inclusion, c'est-à-dire le développement d'usages parfois élaborés sur le plan des manipulations mais ne permettant pas pour autant de négocier une position sociale valorisante au sein des univers sociaux fréquentés (Granjon, 2005, p. 67).

Ainsi révélée à partir de ces strates, la fracture numérique apparaît dès lors résulter de facteurs hétérogènes et interdépendants, au carrefour des sphères technique, culturelle et sociale. Les approches orientées sur ces problématiques posent in fine la question des écarts et des différences (« fracture à réduire », "fossé à combler »), avec, en écho, la problématique des inégalités, problématique qui est loin d'être socialement neutre. Pourtant, qui dit "différence " ou «écart » ne dit pas nécessairement "inégalité ». Pour qu'une différence soit perçue comme inégalité, c'est bien dans un espace social et en référence

à un cadre normatif au nom duquel la dissimilitude constatée est envisagée comme allant à l'encontre de principes de justice et au premier chef, de celui d'égalité. Une inégalité est donc une injustice, c'est-à-dire un fait perçu comme illégitime, 


\section{Nathalie PINĖDE}

en non-adéquation avec un système positif de valeurs qui pousse à en faire la critique (Granjon, 2009, p. 22).

Or les discours autour de la fracture numérique, ainsi que les politiques et les initiatives publiques ou privées promouvant l'accès et le développement des usages du numérique ont cet arrière-plan positif d'une inclusion par les techniques.

Dans cette optique, l'hypothèse est faite que la diminution d'une inégalité technologique (concernant l'équipement ou l'accès) appellera inéluctablement la diminution de toutes les autres inégalités (sociales, culturelles, cognitives, pour ne citer qu'elles). Là se cristallise un premier paradoxe, où le fait technologique, présenté comme une évidence, appelle une forme de standardisation, normative. Cela génère simultanément opportunités et risques: opportunités en matière de décloisonnement et d'inclusion dans une sphère publique (celle d'Internet au premier chef), mais risques en matière d'effets d'échelles et de diversités d'ancrages (culturel, social, individuel).

Un deuxième paradoxe émerge, corollairement au premier. La question des inégalités sous-tendue par la problématique de la fracture numérique renvoie de facto à une lecture des problèmes sous un angle dichotomique... Ainsi s'élaborent des duos oppositionnels, au rang desquels la dimension géographique et territoriale n'est pas des moindres. Mais d'autres dualités sont également fréquemment dénoncées dans cette approche de la fracture numérique. Ainsi, les critères d'âge, de genre, de catégories socioprofessionnelles, d'appartenance ou non au monde du travail sont-ils souvent avancés et créent des lignes de démarcation et une vision antinomique des usages du numérique.

Les questions de l'inégalité et de l'exclusion appelées par cette métaphore de la «fracture » renforcent donc inévitablement cet effet de cristallisation sur une lecture duale, alors même que de nombreuses études (Akrich, 2003 ; Crang, Crosbie et Graham, 2006 ; Hargittai, 2002 ; etc.) montrent judicieusement la complexité des interrelations qui s'établissent au cœur de ces questions et la nécessité d'échapper à des caractérisations sommaires. 


\section{Penser le numérique au prisme des situations de handicap : enjeux et paradoxes de l'accessibilité}

Outre les questions de l'inégalité et de l'exclusion, qui renvoient toutes deux à un positionnement relatif à une norme, d'autres dimensions sociales peuvent être associées à la problématique de la fracture numérique et, parmi celles-ci, la notion de vulnérabilité. Étymologiquement, être vulnérable, c'est la possibilité d'être blessé, d'être exposé aux coups : la référence au corps est alors explicite. Sur le plan social, la vulnérabilité « se présente comme une expérience influençant négativement la capacité d'agir des individus, leur capacité à créer des situations socialement valorisées (réussite, autonomie, responsabilité) pour s'intégrer pleinement à la société » (Brotcorne et al., 2010, p. 64). La vulnérabilité est ici le fait d'une situation individuelle, qui sera vécue du côté des capacités, la capacité d'action dépendant des possibilités de mobilisation de compétences individuelles face aux opportunités de l'environnement (Sen, 2000). C'est dans cette rencontre capacités/opportunités, notamment du point de vue du numérique, que se jouera le degré de conversion en avantage effectif.

Le rapport du handicap au numérique renvoie à ces différents niveaux de fracture et de mise en vulnérabilité, qui peuvent effectivement limiter les possibilités et donc limiter le potentiel de gratification et de reconnaissance en découlant. Ainsi la fracture numérique peut-elle se retrouver dans ce cadre aux trois stades évoqués précédemment: fracture instrumentale, premier niveau, qui sera celle de l'impossibilité/la difficulté (physique ou sensorielle) d'accès via les supports et les interfaces à des contenus et des services; fracture de second degré (méthodologique) résultant des difficultés d'accès, qu'ils soient de l'ordre du tangible ou du cognitif ; enfin, fracture sociale, troisième niveau et conséquence directe de ces empêchements multiples. En miroir de ces fractures se fabriquent des niveaux symétriques de vulnérabilité et de fragilité : vulnérabilité du corps dans le faceà-face avec les dispositifs matériels et sensibles du numérique, face-à-face qui suppose un certain degré d'engagement; vulnérabilité cognitive face à des contenus et des agencements complexes, qui se révèlent porteurs d'exclusion; enfin, vulnérabilité sociale et éducative, effet rebond qui accompagne trop souvent les situations de handicap. Ainsi s'élaborent pour ces 


\section{Nathalie PINÈDE}

personnes les conditions d'une "fracture numérique ", entendue dans une vision extensive et polymorphe, avec tout ce que cela entraîne de risques en matière d'inégalités et d'exclusion. Face à ces risques, la problématique de l'accessibilité (au sens général du terme) et de l'accessibilité numérique, en particulier, constitue une réponse majeure, non dénuée toutefois de certains paradoxes.

\section{De l'accessibilité à l'accessibilité numérique}

Qu'entend-on par ce terme d'accessibilité ? Tout d'abord, il est important de préciser que l'accessibilité ne se réduit pas à l'accès : l'accessibilité rend possible et facile l'accès, que cela soit à des bâtiments physiques, à des ressources informationnelles, des services administratifs en ligne, etc. Ainsi que le rappelle Beauchamps (2009), l'accessibilité a tout d'abord été envisagée du côté du déplacement physique et s'est trouvée de facto corrélée à la notion de distance (et donc à une inscription dans l'espace). Cependant, l'accessibilité s'est rapidement enrichie d'autres dimensions, liées à une approche davantage qualitative, ce qui a modifié sa représentation: dans cette perspective, la focalisation ne s'effectue plus uniquement dans un rapport métrologique au lieu, mais privilégie la perception par les personnes, autorisant ainsi une modulation différenciée en fonction de chacun. Dès lors, le terme d'accessibilité se retrouve souvent mobilisé dans le champ des politiques publiques en rapport avec le handicap, non sans une dynamique évolutive de la prise en compte même de la personne en situation de handicap. Ainsi, Larrouy (2011) montre-telle, dans ses travaux sur l'accessibilité, comment, de la loi d'orientation de $1975^{6}$ à la loi sur l'égalité des droits et des chances de 2005, s'est opéré, en trente ans, un changement sociétal majeur. On passe en effet d'une vision d'une accessibilité restreinte aux personnes handicapées (soit une approche par les déficiences) à une accessibilité "intégrée », revendiquant l'autonomie de la personne et reposant sur une vision systémique de l'accessibilité.

\footnotetext{
${ }^{6}$ Loi d'orientation $n^{\circ} 75-534$ du 30 juin 1975 en faveur des personnes handicapées ; loi relative aux institutions sociales et médico-sociales $n^{\circ} 75-535$ du 30 juin 1975.
} 
Penser le numérique au prisme des situations de handicap : enjeux et paradoxes de l'accessibilité

On retrouve ici ce transfert de responsabilité que l'on a vu dans les évolutions du regard autour du handicap: ce n'est plus la personne qui doit s'adapter à son environnement, mais l'environnement qui doit offrir les conditions d'un accès de qualité, pour toutes les personnes, quelles que soient leurs différences et au-delà d'une approche restreinte $\mathrm{au}(\mathrm{x})$ handicap(s). À ce titre, l'accessibilité est bel et bien l'une des composantes d'une société inclusive, en tant que condition nécessaire mais non suffisante, car il est nécessaire, d'une part, d'avoir une vision globale des enjeux, mais aussi, d'autre part, de ménager la possibilité d'une liberté de choix, en dehors d'une injonction (paradoxale) à l'inclusion (Zaffran, 2015).

Qu'en est-il, dès lors, de l'" accessibilité numérique " comme déclinaison et extension de l'accessibilité, dont nous avons vu qu'elle n'était pas si neutre et évidente que cela? De façon similaire, l'accessibilité numérique ne va pas sans quelque ambiguïté... Dans le cas des écosystèmes numériques, cette perception de la personne en situation de handicap permet de penser autrement conception, dispositifs et usages, en posant la question de la restriction de participation. D'une façon générale (incluant le numérique), on peut définir l'accessibilité comme consistant «à fournir un accès égal aux environnements physiques et numériques, en offrant des lieux et des ressources sûrs, sains, et adaptés à la diversité des personnes susceptibles d'en faire usage » (Folcher et Lompré, 2012, p. 89-90). On peut établir un parallèle avec l'accessibilisation dont il est question pour l'environnement éducatif (Ebersold, 2014) et parler de mise en accessibilité de l'environnement numérique, soit « l'élimination des limitations d'activités ou de restriction de participation subie par une personne » (Folcher et Lompré, 2012, p. 90). Dans le cas de l'accessibilité du web, Tim Berners-Lee rappelle que le " pouvoir du web est dans son universalité " et que "le web est fondamentalement conçu pour fonctionner pour toutes les personnes, quel que soit leur matériel, leur logiciel, leur langue, leur culture, leur emplacement ou leur capacité physique ou 


\section{Nathalie PINÈDE}

mentale $^{7}$ ». Présentée de cette façon, la question de l'accessibilité numérique dépasse des aspects strictement techniques ou fonctionnels liés aux interactions homme-machine pour s'inscrire dans une vision, voire un idéal, d'égalité entre les humains, transcendant la variété (et l'inégalité) -physique, cognitive, géographique, environnementale, etc. - de leurs situations.

On peut néanmoins distinguer deux approches de l'accessibilité numérique: l'accessibilité par le numérique et l'accessibilité du numérique. Bien entendu, ces deux versants de l'accessibilité numérique ne sont ni contradictoires ni exclusifs l'un de l'autre. Ils sont complémentaires et symétriques d'une vision de l'accessibilité liée au numérique qui suppose possibilité d'accès aux environnements numériques, mais aussi résolution de problèmes et diminution de certaines contraintes.

La première approche, celle de l'accessibilité par le numérique, considère le numérique et les TNIC (technologies numériques d'information et de communication) comme des occasions permettant d'accéder à des ressources ou des services apportant une plus-value dans une situation d'empêchement ou de limitation. C'est par exemple la position de Beauchamps (2009), lorsqu'elle effectue un rapprochement entre accessibilité numérique et «mobilité virtuelle» (p.112). On voit également émerger cette vision dans l'article de Marquet (2016), lorsqu'il analyse des projets innovants ${ }^{8}$ visant à résoudre des problèmes d'accessibilité par le numérique. Trois types de représentation émergent à partir des services innovants proposés: l'amélioration de l'accès à l'information, représentation qui domine largement ; la facilitation de la communication interpersonnelle, présente dans une moindre mesure; enfin, la production et l'utilisation de données, qui n'apparaissent que de façon très minoritaire dans les projets liés à l'accessibilité.

\footnotetext{
${ }^{7}$ Traduction libre de : "The power of the Web is in its universality » et " the Web is fundamentally designed to work for all people, whatever their hardware, software, language, culture, location, or physical or mental ability » (https://www.w3.org/standards/webdesign/accessibility).

${ }^{8}$ En l'occurrence, il s'agissait d'un hackaton proposé par la société Transilien pour améliorer la mobilité de tous.
} 
Penser le numérique au prisme des situations de handicap : enjeux et paradoxes de l'accessibilité

Ce versant de l'accessibilité numérique, en tant que réponse par des services et des outils numériques à la problématique globale de l'accessibilité (y compris l'accessibilité physique), s'inscrit dans le registre des technologies dites "capacitantes" (enabling technologies). L'autre versant de l'accessibilité numérique, soit l'accessibilité $d u$ numérique, consiste à faire en sorte que les interfaces, les outils et les contenus puissent être consultables et manipulables par des personnes en situation de handicap (visuel, auditif, moteur, cognitif, par exemple) et donc à agir sur les freins pouvant faire des TIC des technologiques "incapacitantes" (disabling technologies). Dans ce cadre, l'accessibilité numérique concerne principalement les sites web, les services numériques quels qu'ils soient, les applications sur smartphone, les documents numériques, relatifs à des logiciels, des standards et des supports diversifiés. On peut opérer une distinction, à la suite de Boucher (2011) et dans le cadre spécifique de l'accès au numérique, entre l'accessibilité physique et l'accessibilité technologique. L'accessibilité physique renvoie ici à la dimension perceptible du site web ou du document numérique pour tous; l'accessibilité technologique, quant à elle, suppose l'accès indépendamment du matériel utilisé (système d'exploitation, téléphone mobile, etc.). À ces dimensions techniques de l'accessibilité numérique permettant de lever l'obstacle de la perception il faut bien entendu adjoindre l'accessibilité aux contenus et aux savoirs, qui, certes, suppose un accès par les sens (niveau sensoriel), mais implique également le sens (production de significations)... C'est plus spécifiquement cette question de l'accessibilité du numérique, dans ses différentes facettes, que nous allons aborder à partir d'une panoplie de modes d'intervention.

\section{Cadres d'action pour l'accessibilité numérique}

L'accessibilité numérique s'inscrit donc comme un droit pour les personnes en situation de handicap, le droit d'accéder et de participer, sous peine de renforcement des exclusions. À ce titre, la question des normes, problématique fondamentale pour la communication au sein des réseaux numériques (Perriault et Vaguer, 2012), s'impose comme un élément clef dans la mise en 


\section{Nathalie PINÈDE}

œuvre de l'accessibilité, que cela soit pour l'accessibilité physique, numérique ou web (Altinier et Burger, 2012). Au niveau international, le $\mathrm{WCAG}^{9}$, proposé par le W3C (World Wide Web Consortium), fait autorité en matière de référentiel international d'accessibilité numérique pour les contenus web, soit les modalités techniques permettant de diffuser des contenus selon des normes satisfaisant aux critères d'accessibilité. Une première version de ces recommandations, le WCAG $1.0^{10}$, a été proposée en mai 1999, suivie d'une deuxième version en décembre 2008, le WCAG $2.0^{11}$ (et Snow-Weaver, 2008), permettant de prendre en compte les évolutions du web et des formats associés. Quatre principes clefs pour douze règles structurent cette norme ${ }^{12}$ pour l'accessibilité de l'information et des fonctionnalités web :

- perceptible (l'information et les composants de l'interface utilisateur doivent être présentés à l'utilisateur de façon à ce qu'il puisse les percevoir sur le plan sensoriel) ;

- utilisable (les composants de l'interface utilisateur et de navigation doivent être utilisables) ;

- compréhensible (les informations et l'utilisation de l'interface utilisateur doivent être compréhensibles) ;

- robuste (le contenu doit être suffisamment robuste pour être interprété de manière fiable par une large variété d'agents utilisateurs, y compris les technologies d'assistance).

Ces critères concerneront notamment les images, les tableaux, les couleurs, les scripts, la structuration de l'information, la navigation... Pour donner quelques exemples, dans les cas de déficience visuelle, chaque image porteuse d'informations doit avoir une alternative textuelle pertinente, voire, si nécessaire, une description détaillée, permettant de satisfaire au critère " perceptible », pouvant être lue par un synthétiseur vocal. En ce

\footnotetext{
${ }^{9}$ Web Content Accessibility Guidelines (https://www.w3.org/WAl/intro/wcag.php).

${ }^{10}$ Voir : https://www.w3.org/TR/WCAG10/.

11 Traduction française agréée disponible sur : https://www.w3.org/Translations/WCAG20-fr/\#intro.

${ }^{12}$ Le WCAG 2.0 est devenue, en 2012, la norme ISO ISO/CEI 40500:2012

« Technologies de l'information - Règles pour l'accessibilité des contenus Web (WCAG) 2.0 ».
} 
Penser le numérique au prisme des situations de handicap : enjeux et paradoxes de l'accessibilité

qui concerne la navigation dans un ensemble de pages, il faut proposer au moins deux systèmes de navigation différents (menu de navigation, plan du site ou moteur de recherche), ainsi qu'un fil d'Ariane (utilisable, compréhensible). Dans ce contexte, l'accessibilité se situe sur le plan de l'interaction homme-machine, d'un point de vue notamment ergonomique, " elle vise à améliorer l'accès des utilisateurs au système par le biais de l'interface et des caractéristiques matérielles et environnementales du système " (Brangier et al., 2015, p. 71).

Dès 2003, en France, l'association BrailleNet a proposé AccessiWeb ${ }^{13}$, une méthode d'application et de vérification de conformité à la norme WCAG. Par ailleurs, en 2009, le décret d'application $n^{\circ}$ 2009-546 du 14 mai 2009 de l'article 47 de la loi de février 2005 a proposé le Référentiel général d'accessibilité pour les administrations (RGAA) ${ }^{14}$, appuyé sur le WCAG et AccessiWeb, qui fixe

les règles techniques, sémantiques, organisationnelles et d'ergonomie que doivent respecter leurs services de communication publique en ligne afin d'assurer aux personnes handicapées la réception et la compréhension de tout type d'information diffusée sous forme numérique, de leur permettre d'utiliser ces services et, le cas échéant, d'interagir avec ces derniers.

Le RGAA est donc un référentiel à dominante technique ,mais aussi une méthodologie de vérification de conformité aux règles internationales pour les services de communication publique.

En 2016, la promulgation de la loi $n^{\circ} 2016-1321$ pour une République numérique ${ }^{15}$ réaffirme ces principes dans le chapitre 3 , "Accès des publics fragiles au numérique ", en y rajoutant de nouvelles obligations pour les administrations: l'élaboration d'un schéma pluriannuel de mise en accessibilité, l'affichage sur la

\footnotetext{
${ }^{13}$ La version actuelle est celle AccessiWeb 2.2 pour une méthode de vérification de conformité aux WCAG des services web. Voir :

http://www.accessiweb.org/index.php/accessiweb_2.2_liste_generale.html.

${ }_{14}^{14}$ RGAA : https://references.modernisation.gouv.fr/rgaa-accessibilite/. Depuis 2017, la version courante du RGAA est la version 3 .

${ }^{15}$ Disponible sur : https://www.legifrance.gouv.fr/eli/loi/2016/10/7/ECFI1524250L/jo.
} 


\section{Nathalie PINÈDE}

page d'accueil de leurs sites de la mise en conformité avec les règles d'accessibilité (sous peine de sanctions financières), ainsi que la prise en compte de tous les supports (web, intranet, applications mobiles, etc.) pour l'accès à l'information. À noter également l'extension des obligations de mise en accessibilité numérique web pour les sites délégataires de service public et les entreprises au-delà d'un certain chiffre d'affaires.

La même année, la directive européenne 2016/2102 du Parlement européen et du Conseil du 26 octobre 2016 relative à l'accessibilité des sites internet et des applications mobiles des organismes du secteur public ${ }^{16}$ affirme ces principes, présents dès 2010 dans la Stratégie numérique pour l'Europe ${ }^{17}$, en les inscrivant également dans le Plan d'action européen 2016-2020 - Accélérer la mutation numérique des administrations publiques de I'UE ${ }^{18}$. La directive 2016/2102 recommande de s'appuyer sur la norme européenne $301549 \mathrm{~V} 1.1 .2^{19}$ en tant que norme d'accessibilité applicable s'appuyant largement sur les principes de la version 2 du WCAG.

Ainsi qu'on peut le voir à travers ces exemples, différents cadres de régulation, normatifs et réglementaires, à différentes échelles, tous inscrits dans une volonté sociale inclusive, sont présents, notamment par rapport au développement de l'administration et des services publics en ligne. Et c'est une préoccupation légitime, car

si le numérique améliore la qualité des services administratifs, inversement, la disparition du guichet ou le passage complet aux services en ligne font naître un risque de «fracture numérique » entre les individus connectés et les

\footnotetext{
${ }^{16}$ Disponible sur :

https ://eur-lex.europa.eu/legal-content/FR/TXT/?uri=CELEX:32016L2102.

17 Disponible sur : https://eur-

lex.europa.eu/legalcontent/FR/TXT/?uri=celex:52010DC0245R(01) \#footnoteref48. Voir

notamment le chapitre 2.6 « Favoriser les cultures, les compétences et l'intégration numérique ».

${ }^{18}$ Disponible sur :

https://eur-lex.europa.eu/legal-content/FR/TXT/?uri=LEGISSUM:4301896.

${ }^{19}$ Disponible

sur:https://www.etsi.org/deliver/etsi_en/301500 301599/301549/01.01.02 60/en_301549

v010102p.pdf.
} 
Penser le numérique au prisme des situations de handicap : enjeux et paradoxes de l'accessibilité

autres. L'accessibilité des services publics est essentielle pour l'égalité réelle des usagers devant le service public (Algan, Bacache-Beauvallet et Perrot, 2016, p. 7).

D'autres moyens d'action accompagnent ces cadres normatifs et réglementaires, que cela soit sur le plan technique, en matière de méthodes de conception ou encore de sensibilisation/formation. D'un point de vue technique, de multiples solutions émergent pour proposer des systèmes et des interfaces adaptés aux différentes situations individuelles et à la variété des contextes d'usages, afin de favoriser la participation de ces publics et, au-delà, de ces personnes, à une société où le numérique est omniprésent. Par exemple, de nombreuses aides techniques à la communication sont proposées pour pallier les difficultés de consultation de contenus et de navigation sur ordinateurs ou téléphones mobiles pour les personnes en situation de handicap (Balin et Gossart, 2015 ; Lespinet-Najib et al., 2011; Truillet et Raynal, 2013) et proposent une compensation aux différents types de handicap sensoriel: interfaces avec synthèse vocale, claviers virtuels, amplificateurs de voix, accès au contenu écrit ou oral d'un message, aide à la compréhension d'un message écrit/oral, etc. Pour le web, de nombreuses méthodes d'adaptation des pages pour les personnes en situation de handicap visuel existent (par exemple, Bonavero et al., 2016). Mais il serait vain et peu pertinent de tenter ici un recensement exhaustif de toutes ces solutions, les innovations dans le domaine étant nombreuses, notamment en recherche.

L'approche par les méthodes de conception, telles les méthodologies de conception centrées utilisateur $(\mathrm{CCU})^{20}$, prenant en compte des principes de design universel (Choi, 2005) ainsi que l'expérience utilisateur (ou UX Design) (Daumal, 2015; Garrett, 2011), dessine une autre voie opérationnelle pour la mise en œuvre de l'accessibilité numérique. L'intérêt de ces approches est de traduire dans le processus de mise en œuvre ce souci de

${ }^{20}$ Voir notamment les normes ISO 13407:1999 « Processus de conception centrée sur l'opérateur humain pour les systèmes interactifs » et ISO/TR 16982:2002 « Ergonomie de l'interaction homme-système - Méthodes d'utilisabilité pour la conception centrée sur l'opérateur humain ». Pour une synthèse des méthodologies mobilisables dans la mise en application de ces normes, voir Lespinet-Najib (2013). 


\section{Nathalie PINÈDE}

penser ensemble conception et usage, à partir d'une figure de l'usager plus tangible, mais également multiple dans ses attentes et ses besoins.

Enfin, de nombreuses initiatives en matière de formation et de sensibilisation sont déployées, notamment dans un cadre associatif ou de recherche et principalement à propos des normes. En effet, l'exhaustivité descriptive, visée par les normes, se révèle souvent difficile d'accès dans sa forme, et donc sur le fond. Si les normes apparaissent comme d'indispensables régulateurs sociaux, elles ne sont guère utilisables ex abrupto et il peut être intéressant de mettre en lumière ses principes et ses règles clefs en se positionnant du point de vue du concepteur (de services, de contenus), d'autant que ce dernier peut s'avérer néophyte sur le sujet de l'accessibilité (notamment lorsqu'il s'agit de la conception de documents numériques, tels qu'ils peuvent être produits en abondance dans un contexte pédagogique ou organisationnel). Néanmoins, quels que soient les efforts réalisés en matière de simplification orientée utilisateur des dispositifs ${ }^{21}$ (tant sur la forme que sur le fond), demeure d'une part la difficulté de leur appropriation et, d'autre part, de leur intégration dans les pratiques courantes de conception numérique.

\section{Un jeu complexe entre médiations et paradoxes}

En guise de mise en perspective, plusieurs points méritent d'être soulignés, non seulement sur le rôle de l'accessibilité numérique, mais aussi sur les imaginaires et les paradoxes qui l'accompagnent inévitablement.

En procédant à une adaptation différenciée des interfaces en fonction des types de handicap pour permettre d'accéder à des contenus, des ressources et des services, l'accessibilité consiste en une intervention sur les trois fonctions principales des supports (Mitropoulou et Pignier, 2014; Pignier et Drouillat, 2008):

\footnotetext{
${ }^{21}$ Dans le cadre du projet de recherche « Fractures corporelles, fractures numériques » (dirigé par N. Pinède et $\mathrm{V}$. Lespinet-Najib), un « kit d'accessibilité numérique » a été développé et propose actuellement deux modules sur l'accessibilité des documents Powerpoint (https://fracturesnumeriques.fr/kit-accessibilite-powerpoint/) et PDF (https://fracturesnumeriques.fr/kit-accessibilite-pdf/).
} 
Penser le numérique au prisme des situations de handicap : enjeux et paradoxes de l'accessibilité

matérielle (volume, poids, maniabilité) ; formelle (organisation dans l'espace); ergodhique (fonctionnalités et modes d'interaction gestuelle). Dès lors, l'accessibilité numérique, notamment à travers ses principes forts de navigabilité, de pertinence et de clarté, d'information perceptible, lisible et intelligible à tous, est indéniablement instrument de médiation, médiation matérielle, mais aussi médiation des contenus. Par médiation, on comprendra ici une forme de négociation entre plusieurs pôles et entités, dans une situation marquée par des formes d'asymétrie ; la médiation tire alors pour partie sa substance et son essence des interstices qui se créent dans des entre-deux dissymétriques, participant à la création de liens (multiples), ainsi qu'à la construction du sens.

Dans une vision élargie, on peut même dire que l'accessibilité est à la fois médiation et médiatisation (au sens de Peraya, 2008), et ce, afin de faciliter l'appropriation des contenus pour tous les usagers. Par médiatisation, il est entendu tout ce qui concerne la mise en forme relative à un média, soit "le processus de conception et de mise en œuvre de tels dispositifs de formation et de communication médiatisée » (Peraya, 2008, s. p.), ce qui nous entraîne dès lors du côté de l'ingénierie et du design. Les démarches de conception impliquant l'usager, telles que la conception centrée utilisateur (CCU), participent d'une médiatisation prenant en compte les singularités et les différences des publics concernés. Par médiation, il est par contre question de la compréhension "des effets des dispositifs médiatiques, des instruments sur les comportements cognitifs et relationnels " (Peraya, 2008, s. p.). Plus particulièrement, dans le cadre de la médiation instrumentale, l'auteur propose cinq formes de registre : sémio-cognitif (élaboration des significations); sensorimoteur (comportements gestuels); praxéologique (conditions de réalisation de l'action); relationnel (relations entre les sujets impliqués); réflexif (impact sur le sujet). Indéniablement, l'accessibilité numérique consiste en une médiation instrumentale, dans laquelle les registres sensorimoteurs et praxéologiques, en lien aux trois autres registres, prennent une saillance particulière.

Au-delà de cette dimension médiatrice, l'accessibilité numérique peut aussi être considérée comme remédiation, remédiation que l'on peut décrypter selon deux perspectives. La première met en 


\section{Nathalie PINĖDE}

œuvre de nouvelles formes de réécriture et de rematérialisation (que cela soit sur les plans de la syntaxe, de la forme communicationnelle [écrit, oral], ou encore de l'architecture informationnelle). Par exemple, en ce qui concerne les personnes malvoyantes ou non voyantes, l'accessibilité numérique suppose pour les images une réinscription scripturale, pouvant être convertie sur le plan sonore grâce à des logiciels de synthèse vocale, ce qui influe inévitablement sur la production des significations pour l'usager. "Même si l'on ne peut raisonnablement pas réduire un message au moyen matériel de sa transmission, tout changement dans le système des communications a nécessairement d'importants effets sur les contenus transmis " (Goody, 1979, p. 46). Dans une deuxième perspective, il s'agit de remédiation au sens de " réparer, pallier », donc de remédiation par l'apport d'une forme de soin (care) pour des utilisateurs du numérique empêchés sur un certain nombre de plans.

Néanmoins, au-delà de cette fonction médiatrice plurielle de l'accessibilité numérique, plusieurs paradoxes peuvent être singulièrement relevés, à différents niveaux. Tout d'abord, les constats en ce qui a trait aux réalités de terrain restent mitigés sur un certain nombre de points, alors même qu'un appareil réglementaire et normatif conséquent a été instauré depuis plus d'une douzaine d'années et que de nombreuses initiatives en matière d'accompagnement, de sensibilisation ou d'innovation sont déployées, notamment dans un cadre associatif ou de recherche. Ainsi, à la suite de la publication du décret proposant le RGAA, obligation était faite aux sites web publics et administratifs de se mettre en conformité avec ces règles d'accessibilité dans un délai de deux à trois ans, obligation qui n'a cessé d'être reportée depuis. Cinq ans après la promulgation du RGAA, en 2014, une enquête menée par l'association BrailleNet sur 600 sites web publics français $^{22}$ montrait que seuls $4 \%$ d'entre eux répondaient aux normes d'accessibilité. D'autres enquêtes (Lazar, DudleySponaugle et Greenidge, 2004 ; Lespinet-Najib et al., 2015) ont mis à jour les préjugés persistants du côté des concepteurs de

${ }^{22}$ Voir : http://www.braillenet.org/documents/communique-braillenet-2014-03-25.pdf. 
Penser le numérique au prisme des situations de handicap : enjeux et paradoxes de l'accessibilité

sites web : coût de réalisation plus élevé, plus grande pauvreté graphique ou plus grande difficulté de mise en œuvre, et ce, alors même que les apports de l'accessibilité numérique pour tous (navigation plus aisée dans le site, meilleur référencement, etc.), au-delà des usagers que sont les personnes en situation de handicap, ont été à maintes reprises démontrés (Adam et Kreps, 2009). Si certains secteurs, comme celui des bibliothèques, font montre d'un véritable dynamisme en la matière (Lompré, 2017 ; Maisonneuve et Lenepveu, 2017 ; Papy et Jakubowicz, 2018), il reste encore un travail important à réaliser. En matière d'incitation/obligation, alors que la directive européenne 2016/2102 avait fixé le 23 septembre 2018 comme date butoir pour s'y conformer et qu'en France, l'obligation par le RGAA existe depuis dix ans, l'accord-cadre 2018-2020 de l'État «Accessibilité numérique et conformité au RGAA » devrait donner une impulsion forte au processus de mise en accessibilité des sites web, des applications métiers et des applications mobiles des services publics. Au-delà de ces initiatives réglementaires persiste néanmoins la nécessité de sensibilisation, d'éducation et de formation (Bevan et Spinhof, 2007) auprès de publics diversifiés, et notamment des concepteurs (actuels ou futurs) : concepteurs de sites et d'applications web, mais aussi concepteurs de contenus et de documents, quel que soit le domaine concerné (universitaire, administratif, industriel, commercial, etc.), ou encore apprentis concepteurs dans les filières de formation dédiées.

Par ailleurs, les éléments positifs relatifs à une démarche d'accessibilité numérique à visée inclusive restent assortis d'effets ambivalents. Subsistent ou se développent d'inévitables formes de complexité, sur le plan de la conception des interfaces et des services ou des processus info-communicationnels. Ainsi, quels que soient les efforts réalisés en matière de contenus accessibles et d'encadrement réglementaire, à l'heure de la numérisation du nombre de services, pour les démarches administratives standard ou la gestion des droits sociaux, les risques de marginalisation supplémentaires sont réels, que cela soit pour les publics en situation de handicap ou les publics socialement défavorisés. " La dématérialisation entraîne une forme de structuration et de rationalisation des offres, qui se heurte à la complexité des 


\section{Nathalie PINÈDE}

parcours heurtés et précisément "hors des cases" des publics cibles et de leurs difficultés d'appropriation » (Alberola, Croutte, et Hoibian, 2016, p. 35). D'autres études confirment ces difficultés, par exemple sur les sites de réservation en ligne type "SNCF " (Benassaya et al., 2017). La complexité croissante des environnements numériques, multipliant les niveaux de services, les strates d'information et les modalités d'interactions, ne va évidemment pas dans le sens d'une facilitation pour les usagers en situation de handicap. Ce à quoi on peut rajouter qu'il n'y a pas nécessairement corrélation entre le degré d'accessibilité des sites (soit respectant les normes d'accessibilité) et l'utilisabilité ${ }^{23}$ perçue par les utilisateurs (Aizpurua, Harper et Vigo, 2016 ; Lompré, Folcher et Gouedard 2008; Petrie et Kheir, 2007). En effet, l'entrée par la conformité aux règles ne recouvre pas l'intégralité de l'expérience utilisateur ${ }^{24}$ et, dans cette perspective, le respect des normes de type WCAG et RGAA est nécessaire mais non suffisant. Car au-delà d'une norme " standardisant " par essence des attendus, c'est bien la question des usages au sens large qui se pose, dans ce rapport expérientiel aux dispositifs inscrits dans un environnement, rapport toujours singulier et multidimensionnel.

D'autre part, il ne faut pas sous-estimer la difficulté du processus de traduction propre à l'accessibilité numérique. Prendre en compte la réalité des personnes et de leur situation de handicap impose de prendre en charge leur complexité (corporelle, sensorielle, cognitive, émotionnelle, etc.) dans les interactions avec des dispositifs et des interfaces numériques. L'accessibilité numérique a pour dessein de "parler " au plus grand nombre, et idéalement à tous, ce qui relève d'une nécessaire universalité. Or cela ne peut finalement s'accomplir sans un aplanissement de la densité informationnelle, voire de la richesse esthétique, présente sur nombre d'interfaces. Comment convertir une communication sensible, visuelle en signes perceptibles en fonction du handicap sans en "trahir » le contenu ? Comment donner à voir, à lire, à entendre, à s'émouvoir et à comprendre, pour tout un chacun, à

\footnotetext{
${ }^{23}$ Par utilisabilité, on entendra la qualité et l'efficacité des interactions pour la réalisation d'une tâche, à moindre effort.

${ }^{24}$ Par expérience utilisateur, on entendra ici le croisement de plusieurs critères (d'après Brangier et Bastien, 2010) : l'accessibilité, l'utilisabilité, l'émotionnalité et l'influençabilité.
} 
Penser le numérique au prisme des situations de handicap : enjeux et paradoxes de l'accessibilité

travers un accès qui préserve la capacité et la liberté de mises en significations plurielles? Ce sans compter que l'accessibilité numérique contient ses propres inégalités: si, dans le cadre du handicap visuel, bon nombre de ressources existent, le handicap mental ou les troubles cognitifs ${ }^{25}$ sont moins pris en charge, l'opération de traduction et de réécriture, plus complexe, devant porter ici non seulement sur la dimension perceptible (espacement des termes, organisation visuelle du texte, etc.) mais aussi sur la formulation des contenus.

Enfin, nous terminerons par l'ambivalence sociale associée à l'accessibilité numérique. Les approches visant à analyser les dispositifs numériques à l'aune des usages des personnes en situation de handicap, notamment par le développement de normes visant à lutter contre les risques d'exclusion, mais aussi par la promotion - légitime - de la conception par et avec l'accessibilité numérique, participent à leur manière au paradoxe de l'inclusion que nous avons évoqué en première partie. L'accessibilité constitue une réponse à un paradigme positiviste du numérique, désigné comme pouvant être exclusif : la recherche de l'intégration par la défense de cette forme d'égalité suppose implicitement l'acceptation et la légitimation de son inéluctabilité sociale. Pour autant, comment ne pas devoir jouer avec les règles en vigueur? La contrainte technique se pose

en tant que mode d'inscription du social, puisque les scripts des dispositifs techniques ne sont pas seulement ce qui rend éventuellement possible la réalisation de soi, mais aussi ce qui peut témoigner de son impossibilité et même ce qui peut participer de sa limitation et de son empêchement (Granjon et Denouel, 2011, p. 15).

On ne peut donc passer sous silence un arrière-plan ambigu associé à l'accessibilité numérique. Penser autrement le rapport société/numérique relève d'un effort de mise à distance permanent. Si la cause est juste, et elle l'est indubitablement, car l'on ne saurait dénier à certains (et donc à tous) le droit d'accéder par la technique à des ressources multiples (informationnelles,

${ }^{25}$ Notamment ceux que l'on appelle « troubles dys » : dyslexie, dysorthographie, etc. 


\section{Nathalie PINÈDE}

communicationnelles, etc.), les moyens et les argumentations déployés entretiennent une forme d'inéluctabilité, pouvant aller jusqu'aux mythologies du posthumain.

Les technologies créent de la démesure. S'il s'agit de les maîtriser, on n'évitera pas d'en brider les prétentions. Comment ? [...] Sur le front des mentalités, en contribuant à développer un autre imaginaire que celui des utopies posthumaines: un imaginaire qui fera droit à des valeurs soucieuses de préserver la vulnérabilité et la conscience de la fragilité comme le véritable privilège de l'humain (Besnier, 2013, p. 51).

II s'agit donc de garder raison et de conserver un nécessaire regard critique permettant d'aborder la question des fragilités et des vulnérabilités, notamment dans cette articulation entre les situations de handicap et les usages du numérique, comme un enjeu tout à la fois majeur et complexe.

\section{Conclusion}

Entreprenant une approche critique du paradigme des usages, Voirol (2011) identifie une crise de celui-ci, crise issue selon lui de trois oublis principaux: oublis de la "vulnérabilité des sujets", «de la contrainte technique » et « de la critique». La problématique handicap/numérique illustre avec acuité ces zones grises. Les fragilités sensorielles, physiques, voire cognitives, de ces personnes, auxquelles se surajoutent souvent des difficultés sociales, sont généralement ignorées. Or le phénomène majeur de vieillissement de la population, les aléas et les accidents de vie sont autant de facteurs, au-delà des déficiences natives, pouvant nous mettre en situation de handicap face au numérique. En contrepoint, l'image sous-jacente, tel un acquis, d'un « cela va de soi » ou d'un " usager numérique idéal » est un fantasme, qui sous-tend pourtant encore nombre de réalisations et de conceptions. La réalité des personnes et des pratiques est infiniment plus diversifiée qu'une majorité de cas "moyens" observables. Les ajustements à la technique sont permanents et concernent tous les usagers, mais pour ceux qui sont empêchés, 
Penser le numérique au prisme des situations de handicap : enjeux et paradoxes de l'accessibilité

quelle qu'en soit la raison, cette nécessaire adaptation implique de surcroît le recours indispensable à des couches médiatrices supplémentaires, s'incarnant dans des formes variées (aides et alternatives, techniques ou humaines).

La problématique de l'accessibilité numérique et des situations de handicap soulève donc des questions complexes, à différentes échelles, ne se réduisant pas aux interactions avec des outils. Si elle mobilise de manière assez évidente et visible des aspects réglementaires, techniques ou normatifs, elle interpelle également les représentations sociales à l'œuvre, notamment dans les rapports des concepteurs de systèmes, des médiateurs de contenus ou des usagers, aux dispositifs sociotechniques et aux processus info-communicationnels qui les accompagnent. En miroir, c'est de façon beaucoup plus large l'articulation numérique/société qui est ici interrogée, mettant dès lors à jour certains paradoxes. La combinaison, toujours singulière, d'hétérogénéités multiples contribue à créer des équilibres, mais aussi des déséquilibres permanents entre l'individu, le social et le numérique. Ainsi, si l'accessibilité numérique est adossée au principe - légitime - de défense de l'égalité, cela n'est pour autant pas exempt d'ambivalences (Le Crosnier et Besnier, 2014). Parmi celles-ci figure en bonne place l'acceptation implicite de l'inéluctabilité des usages du numérique, renforcée par le phénomène d'injonctions généralisées à la participation (Proulx, 2017). À ce titre, si l'accessibilité numérique, pour des personnes fragiles et vulnérables à différents égards, est un droit essentiel dans nos sociétés actuelles, rendre possible l'accès permet aussi d'exercer sa liberté de choisir.

\section{Références}

Adam, A. et Kreps, D. (2009). Disability and discourses of Web accessibility. Information, Communication \& Society, 12(7), 1041-1058.

Akrich, M. (2003). Technologies de communication et genre. Des relations en construction. Réseaux, 4(120), 53-86. 


\section{Nathalie PINÈDE}

Akrich, M., (2010). Comment décrire les objets techniques ?. Techniques \& Culture, 54-55(1), 205-219.Aizpurua, A., Harper, S. et Vigo, M. (2016). Exploring the relationship between web accessibility and user experience. International Journal of Human-Computer Studies, 91, 13-23.

Alberola, E., Croutte, P. et Hoibian, S. (2016). La « double peine » pour des publics fragilisés face au tout-numérique. Annales des Mines - Réalités industrielles, 3, 32-36.

Algan, Y., Bacache-Beauvallet, M. et Perrot, A. (2016). Administration numérique. Notes du conseil d'analyse économique, 34(7), 1-12.

Altinier, A. et Burger, D. (2012). Accessibilité web : normes et bonnes pratiques pour des sites plus accessibles. Paris, France : Éditions Eyrolles.

Ayon, H. S. (2016). E-inclusion des personnes en situation de handicap psychique. Les Cahiers du numérique, 12(1), 133-170.

Balin, P. et Gossart, C. (2015). L'accessibilité des TIC par les personnes handicapées : état des lieux du contexte actuel. Terminal, (116).

Repéré à https://journals.openedition.org/terminal/751

Beauchamps, M. (2009). L'accessibilité numérique : transformer le risque de renforcement des inégalités numériques en opportunité. Les Cahiers du numérique, 5(1), 101-118.

Benassaya, M., Dusaucy, V., Lemarié, J., Oriola, B., Vigouroux, N. et Mojahid, M. (2017, juillet). État des lieux de l'accessibilité de trois sites de réservation de billets de transport. Communication présentée lors de la $9^{\text {ème }}$ Conférence de psychologie ergonomique (Epique 2017), Dijon, France.

Besnier, J.-M. (2013). Quelles utopies à l'ère du numérique ?. Études, 7(419), 43-51.

Bevan, N. et Spinhof, L. (2007). Are Guidelines and Standards for Web Usability Comprehensive?. Dans J. A. Jacko (dir.), HumanComputer Interaction. Interaction Design and Usability. $\mathrm{HCl}$ 
Penser le numérique au prisme des situations de handicap : enjeux et paradoxes de l'accessibilité

2007. Lecture Notes in Computer Science, vol. 4550 (p. 407419). Berlin, Allemagne : Springer-Verlag.

Bonavero, Y., Huchard, M., Meynard, M. et Waffo Kouhoué, A.

(2016). État de l'art des méthodes d'adaptation des pages web en situation de handicap visuel. Dans C. Jouffrais, F. Cabestaing et M. Slimane (dir.), Handicap. Conférence sur les aides techniques pour les personnes en situation de handicap (p. 187-192). Repéré à https://hallirmm.ccsd.cnrs.fr/lirmm-01330727/document

Boucher, A. (2015). Ergonomie web : pour des sites web efficaces. Paris, France : Éditions Eyrolles.

Bouvier, G. (2011). L'enquête Handicap-Santé. Présentation générale. Institut national de la statistique et des études économiques (INSEE), Série des documents de travail de la Direction des statistiques démographiques et sociales, $\mathrm{n}^{\circ} \mathrm{F} 1109$.

Brangier, E. et Bastien, J. (2010). L'évolution de l'ergonomie des produits informatiques : accessibilité, utilisabilité, émotionnalité et influençabilité. Dans G. Vallery, M.-C. Le Port et M. Zouinar (dir.). Ergonomie, conception de produits et services médiatisés (p. 307-328). Paris, France : Presses universitaires de France.

Brangier, E., Desmarais, M., Nemery, A. et Prom Tep, S. (2015). Évolution de l'inspection ergonomique : vers une intégration des critères d'accessibilité, de praticité, d'émotion et de persuasion dans l'évaluation ergonomique. Journal d'Interaction PersonnesSystème, 4(1), 69-84.

Brotcorne, P., Damhuis, L., Laurent, V., Valenduc, G. et Vendramin, P. (2010). Diversité et vulnérabilité dans les usages des TIC. La fracture numérique au second degré. Gent, Belgique : Academia Press.

Buckingham, D. (2016). Defining digital literacy. Nordic Journal Of Digital Literacy, (Special Issue), 21-34.

Casilli, A. (2010). Technologies capacitantes et « disability divide " : enjeux des usages du numérique dans les situations de handicap. Dans J. Gaillard (dir.), Vers la fin du handicap ? 


\section{Nathalie PINÈDE}

Pratiques sportives, nouveaux enjeux, nouveaux territoires (p. 501-515). Nancy, France : Presses universitaires de Nancy.

Choi, S. (2005). Universal Design: A Practical Tool for a Diverse Future. International Journal of the Diversity, 6, 116-124.

Crang, M., Crosbie, T. et Graham, S. (2006). Variable Geometries of Connection: Urban Digital Divides and the Uses of Information Technology. Urban Studies, 43, 2551-2568.

Daumal, S. (2015). Design d'expérience utilisateur : principes et méthodes UX. Paris, France : Éditions Eyrolles.

Dobransky, K. et Hargittai, E. (2006). The Disability Divide in Internet Acces and Use. Information, Communication and Society, 9(3), 313-334.

Ebersold, S. (2014). Accessibilité, politiques inclusives et droit à l'éducation : considérations conceptuelles et méthodologiques. ALTER. European Journal of Disability Research, 9, 22-33.

Flichy, P. (2004). L'individualisme connecté entre la technique numérique et la société. Nouvelles réflexions sur l'internet. Réseaux, 22(124), 17-51.

Folcher, V. et Lompré, N. (2012). Accessibilité pour et dans l'usage : concevoir des situations d'activité adaptées à tous et à chacun. Le travail humain, 75(1), 89-120.

Fougeyrollas, P., Cloutier, R., Bergeron, H., Côté, J. et SaintMichel, G. (1998). Processus de production du handicap. Classification québécoise. Québec, Canda : Réseau international sur le processus de production du handicap.

Garrett, J. J. (2011). Les éléments de l'expérience utilisateur : placer l'utilisateur au cœur de la conception des produits web et mobiles. Paris, France : Pearson Éducation France.

George, E. (2004). La « fracture numérique » en question. Dans E. Guichard (dir.), Mesures de l'Internet (p. 152-165). Paris, France : Les Canadiens en Europe.

Gilster, P. (1997). Digital literacy. New York, NY : Wiley Computer Pub. 
Penser le numérique au prisme des situations de handicap : enjeux et paradoxes de l'accessibilité

Goody, J. (1979). La raison graphique. La domestication de la pensée sauvage. Paris, France : éditions de Minuit.

Granjon, F. (2005). Pour une sociologie critique de la fracture numérique : de quelques éléments programmatiques. Dans L. Vieira et N. Pinède (dir.), Enjeux et usages des TIC : aspects sociaux et culturels (p. 59-68). Bordeaux, France : Presses universitaires de Bordeaux.

Granjon, F. (2009). Inégalités numériques et reconnaissance sociale. Des usages populaires de l'informatique connectée. Les Cahiers du numériques, 1(5), 19-44.

Granjon, F. et Denouel, J. (2011). Communiquer à l'ère du numérique. Regards croisés sur la sociologie des usages. Paris, France : Presses des Mines.

Hargittai, E. (2002). Second-level digital divide: Differences in people's online skills. First Monday, 7(4), University of Illinois at Chicago. Repéré à https://firstmonday.org/ojs/index.php/fm/article/view/942/864

Lamarche, T., Rallet, A. et Zimmermann, J. B. (2006). Les technologies de l'information et de la communication comme réductrices ou amplificatrices des inégalités. Terminal, (95-96), 7-13.

Larrouy, M. (2011). L'invention de l'accessibilité. Des politiques de transports des personnes handicapées aux politiques d'accessibilité (1975-2005). Grenoble, France : Presses universitaires de Grenoble.

Lazar, J., Dudley-Sponaugle, A. et Greenidge, K. D. (2004). Improving Web accessibility. Computer in Human Behavior, 20, 269-28.

Le Crosnier, H. et Besnier, J.-M. (2014). Le numérique peut-il aider à résoudre les inégalités qu'il engendre ?. CIPPA - Séminaires IGEP, 1(5). Repéré à http://cippa.paris-sorbonne.fr/wpcontent/uploads/2013/03/CIPPA-IGEP-2012-2013-05-Lenumérique-peut-il-aider-à-résoudre-les-inégalités-qu'ilengendre.pdf 


\section{Nathalie PINÈDE}

Lespinet-Najib, V. (2013). De la neuropsychologie cognitive à la cognitique : vers une recherche transdisciplinaire (Mémoire d'habilitation à diriger des recherches). Université Bordeaux 1.

Lespinet-Najib, V. et Belio, C. (2013). Classification des handicaps : enjeux et controverses. Hermès, 66, 104-110.

Lespinet-Najib, V., Pinède, N., Belio, C., Demontoux, F. et Liquète, V. (2015). L'accessibilité Web, en 2013, en France : enquête nationale sur les pratiques et les usages des professionnels du Web. Terminal, (116). Repéré à https://journals.openedition.org/terminal/649

Lompré, N. (2017). L'accessibilité numérique. Comprendre l'accessibilité numérique. Dans F. Fontaine-Martinelli et L. Maumet (dir.), Accessibilité universelle et inclusion en bibliothèque (p. 93-100). Paris, France : Association des bibliothécaires de France.

Lompré, N., Folcher, V., Gouedard, C. (2008). Accessibilité des ressources numériques pour la lecture : du respect des critères au point de vue des utilisateurs. Dans N. Vigouroux et P. Gorce (dir.), Handicap'2008 (p. 106-111). Paris, France : Cépaduès Éditions.

Maisonneuve, M. et Lenepveu, P. (2017). Accessibilité numérique. Bulletin des bibliothèques de France (BBF), 11, 16-25.

Marquet, C. (2016). Des services connectés pour améliorer l'accessibilité des gares ?. Espace populations sociétés, (2). Repéré à https://journals.openedition.org/eps/6344\#text

Mitropoulou, E. et Pignier, N. (2014). Introduction. Interroger les supports ? Matières, formes et corps. Communication \& langages, 4(182), 13-28.

Papy, F. et Jakubowicz, C. (2018). Bibliothèque numérique et innovation (vol. 2). Londres, Angleterre : ISTE Éditions.

Peraya, D. (2008). Un regard critique sur les concepts de médiatisation et médiation : nouvelles pratiques, nouvelle modélisation. Les Enjeux de l'information et de la 
Penser le numérique au prisme des situations de handicap : enjeux et paradoxes de l'accessibilité

communication. Repéré à https://archiveouverte.unige.ch/unige:17665

Perriault, J. et Vaguer, C. (2010). La norme numérique. Savoir en ligne et Internet. Paris, France : CNRS éditions.

Petrie, H. et Kheir, O. (2007). The Relationship between Accessibility and Usability of Websites. Proceedings of the SIGCHI Conference on Human Factors in Computing Systems (CHI '07) (p. 397-406). New York, NY : ACM Press.

Pignier, N. et Drouillat, B. (2008). Le webdesign. Sociale expérience des interfaces web. Paris, France : HermèsLavoisier.

Proulx, S. (2017). L'injonction à participer au monde numérique. Communiquer, 20, 15-27.

Reid, L. G. et Snow-Weaver, A. (2008, avril). WCAG 2.0: A web accessibility standard for the evolving web. Proceedings of the 2008 international cross-disciplinary conference on Web accessibility (W4A '08) (p. 109-115). New York, NY : ACM Press.

Rizza, C. (2006). La fracture numérique, paradoxe de la génération Internet. Hermès, 45, 25-32.

Roy, D. (2016). Les personnes âgées et handicapées en France et les politiques publiques d'accompagnement. Revue française des affaires sociales, 4, 21-33.

Saemmer, A. (2017). Lectures préférentielles du dispositif tablette. Dans V. Liquète et $\mathrm{K}$. Soumagnac (dir.), Les affiliations par et avec le numérique (p. 57-72). Paris, France : Hermann.

Sen, A. (2000). Repenser l'inégalité. Paris, France : Seuil.

Soupizet, J. F. (2004). La fracture numérique. Netsuds « Réduire le fossé numérique Nord-Sud, quels enjeux ? », 9.

Stiegler, B. (2010). Pharmacologie de l'épistémè numérique. Dans B. Stiegler (dir.), Digital Studies. Organologie des savoirs et technologies de la connaissance (p. 13-26). Limoges, France : FYP éditions. 
Truillet, P. et Raynal, M. (2013). Conception rapide d'aides techniques pour personnes tétraplégiques. MEI - Médiation et Information, (36), 103-115.

Vendramin, P. et Valenduc, G. (2006). Fractures numériques, inégalités sociales et processus d'appropriation des innovations. Terminal, (95-96), 137-154.

Vitali-Rosati, M. (2014). Pour une définition du « numérique ». Dans M. Erbele-Sinatra Michael, M. Vitali-Rosati (dir.), Pratiques de l'édition numérique (p. 63-75). Montréal, Canada : Les Presses de l'Université de Montréal.

Voirol, O. (2011). L'intersubjectivation technique : de l'usage à l'adresse. Penser une théorie critique de la culture numérique. Dans F. Granjon et J. Denouel (2011), Communiquer à l'ère du numérique. Regards croisés sur la sociologie des usages (p. 127-157). Paris, France : Presses des Mines.

Zaffran, J. (2015). Accessibilité et handicap. Grenoble, France : Presses universitaires de Grenoble. 